\title{
La densidad, riqueza y composición arbóreas definen parches detectados remotamente en una selva subperennifolia
}

\author{
Alejandra del Pilar Ochoa-Franco ${ }^{1}$, José René Valdez-Lazalde ${ }^{1 *}$, \\ Héctor Manuel de los Santos-Posadas ${ }^{1}$, José Luis Hernández-Stefanoni², \\ Juan Ignacio Valdez-Hernández ${ }^{1} \&$ Gregorio Ángeles-Pérez ${ }^{1}$ \\ 1. Postgrado en Ciencias Forestales, Colegio de Postgraduados, Km 36.5 Carr. México-Texcoco, Montecillo, Texcoco, \\ México; ochoa.a8a8@gmail.com, valdez@colpos.mx,hmsantos@colpos.mx, ignaciov@colpos.mx, \\ gangeles@colpos.mx \\ 2. Unidad de Recursos Naturales, Centro de Investigación Científica de Yucatán A.C, Mérida, Yucatán, México; \\ j1_stefanoni@cicy.mx \\ * Correspondencia
}

Recibido 28-VIII-2018. Corregido 08-I-2019. Aceptado 10-VI-2019.

\begin{abstract}
Tree density, species richness, and composition drive vegetation patches identified from remotely-sensed data in a semi evergreen tropical forest. A proposal for characterizing habitat of forests, obtained from an object-oriented classification of RapidEye multiespectral imagery, based on dissimilarity matrices of vegetation structure, species diversity and composition is presented. The study area is a forested landscape mosaic after slash and burn agriculture (Ac: 8-23 years ago), selective logging (Fs: 43-53 years ago), and selective logging and forest fire (Fc: 21-28 years ago). The site is located in the central part of Quintana Roo, México, where three vegetation patches were delineated according to remotely sensed multiespectral imagery. Mean differences between vegetation structure properties of each vegetation patch were obtained through a permutational multivariate analysis of variance $(\mathrm{P}<0.001)$. Species richness, stem density per hectare, and the axis-1 scores of the non-metric multidimensional scaling ordination of specific composition were identified as the vegetation attributes more relevant to differentiate the vegetation patches by a multinomial logistic model. Fc vegetation patch is characterized by the greatest mean values on Shannon-Wiener index, species richness, and stem density. The Fs has the greatest mean values of canopy height, basal area, and biomass at 80 percentile, and the Ac vegetation patch has the lowest values of all mentioned metrics. The species with the greatest relative importance value were: Ac: Bursera simaruba and Piscidia piscipula, Fs: Gymnanthes lucida and Manilkara zapota, Fc: G. lucida and B. simaruba. The uncertainty associated with the metrics assessed by vegetation patch was smaller than the uncertainty of the whole area, because of the efficient variability aggregation of the field data. We conclude that multiespectral information is a reliable tool for distinguishing vegetation patches with specific features, as stem density, specific composition, and species richness.
\end{abstract}

Key words: RapidEye; image segmentation; relative importance value; permanova; multinomial model; tropical forest; habitat characterization.

Ochoa-Franco, A. P., Valdez-Lazalde, J. R., de los Santos-Posadas, H. M., HernándezStefanoni, J. L., Valdez-Hernández, J. I. \& Ángeles-Pérez, G. (2019). La densidad, riqueza y composición arbóreas definen parches detectados remotamente en una selva subperennifolia. Revista de Biología Tropical, 67(4), 692-707.

México se ha unido a los esfuerzos internacionales para la conservación de la biodiversidad (CBD, 2010), a través de una estrategia nacional (CONABIO, 2016) y la integración de la conservación de la diversidad a nivel de hábitat, como elemento relevante en la gestión forestal (ENAIPROS, 2013). Si bien el concepto hábitat dista de pertenecer a la terminología 
científica estándar (Kirk et al., 2018), los conceptos consensuados por la directiva de hábitat en Europa (EEA, 2014) y la nación mexicana, son aplicables para la planificación forestal en aspectos clave como la ordenación forestal y toma de decisiones sobre ciclos de intervención silvícola, o la definición de corredores biológicos. Este enfoque, los considera como unidades del paisaje, naturales o transformadas, caracterizadas por un conjunto de factores que determinan la distribución y abundancia de plantas y animales (Jardel-Peláez, 2015). Su caracterización es ecológicamente importante, puesto que es el primer paso para la definición de nichos ecológicos específicos, fundamental para estudios de distribución de poblaciones y una forma eficiente de evaluar la diversidad potencial de especies en un ecosistema (Garshelis, 2000).

La vegetación tropical ostenta una relevancia innegable para la biodiversidad al albergar más de 43000 especies arbóreas (Slik et al., 2015). Las selvas secas, a pesar de su alta diversidad y endemismo, son las más amenazadas debido a las altas tasas de deforestación, degradación y fragmentación que enfrentan (Miles et al., 2006). Son paisajes dominados por vegetación secundaria que cambia relativamente rápido en el tiempo su composición específica y su estructura debido a perturbaciones humanas o naturales. Las perturbaciones ocasionan cambios en la composición de especies, lo que puede identificarse directamente evaluando el recambio de especies (Chan-Dzul, 2010, Granados-Victorino, Sánchez-González, Martínez-Cabrera, \& Octavio-Aguilar, 2017) o a través de índices de diversidad, equidad, o riqueza (Derroire et al., 2016). Los cambios en estructura son reconocibles en el área basal, la densidad de tallos por hectárea (RománDañobeytia, Levy-Tacher, Macario-Mendoza, \& Zúñiga-Morales, 2014), la altura (Letcher et al., 2015) y la biomasa aérea (Urquiza-Haas, Dolman, \& Peres, 2007). De manera que las dos aproximaciones más comunes para la caracterización del hábitat se basan en la composición de especies (Iqbala, Khanb, Khanc, Ahmadb, \& Ahmadd, 2018) y la estructura de la vegetación
(Sterenczak, Lisanczuk, \& Erfanifard, 2018), las cuales son precisamente las dos clases de variables esenciales de la biodiversidad (EBVs) más importantes para la conservación (Pereira et al., 2013; Asaad, Lundquist, Erdmann, \& Costello, 2016).

La estructura de la vegetación y la composición de especies están estrechamente relacionadas, ya que la estructura tridimensional de los bosques y selvas, es simultáneamente producto y causa de los procesos ecosistémicos $\mathrm{y}$, por lo tanto, de la diversidad taxonómica (Spies, 1998). Investigaciones que contrastan de forma multivariada dichos atributos en diferentes etapas sucesionales (SánchezSánchez, Islebe, \& Valdez Hernández, 2007; Granados-Victorino et al., 2017), historiales de disturbio (Urquiza-Haas et al., 2007, CarreónSantos \& Valdez-Hernández, 2014) o manejo (Gutiérrez-Granados, Pérez-Salicrup, \& Dirzo, 2011; Báez-Vargas et al., 2017), han permitido identificar las variables más sensibles para cada caso, pero típicamente son caracterizaciones basadas en datos de campo. Por otro lado, investigaciones que utilizan sensores remotos permiten el modelado y mapeo del hábitat (Ichter, Savio, Evans, \& Poncet, 2017), no obstante, aunque existen aproximaciones multivariadas a través de índices (Pasher \& King, 2010; Hakkenberg, Peet, Urban, \& Song, 2018a), o variable por variable (Gallardo-Cruz et al., 2012; Solórzano, Meave, Gallardo-Cruz, González, \& Hernández-Stefanoni, 2017) frecuentemente se enfocan en una o dos variables elegidas a priori como biomasa y riqueza de especies (Hernández-Stefanoni, Dupuy, TunDzul, \& May-Pat, 2010) o índices de diversidad (George-Chacón, 2017).

Dado que la información derivada de datos espectrales, recolectados mediante sensores remotos, puede actuar como proxy de los atributos de la estructura de la vegetación y la composición de especies (Lausch et al., 2016) al reflejar su heterogeneidad interna (GallardoCruz et al., 2012), recientemente se ha abordado la delimitación de parches de vegetación a través de clasificaciones orientadas a objetos con datos multiespectrales (Fujiki, Okada, 
Nishio, \& Kitayama, 2016), en combinación con datos LiDAR (Machala \& Zejdová, 2017), o con algoritmos de clasificación (Liu, Gong, $\mathrm{Hu}$, \& Gong, 2018). Particularmente para el seguimiento de los paisajes con aprovechamiento maderable, es conveniente identificar las variables que más distinguen los parches de vegetación con edades o historiales distintos, previo a la creación de índices o modelos espaciales, para entender, conservar y aprovechar mejor los paisajes tropicales, facilitando la focalización de recursos, en el marco de las certificaciones de manejo sostenible de las selvas.

Este trabajo tuvo como objetivo caracterizar parches de vegetación en un paisaje de bosque tropical seco, a través de tres matrices de disimilitud del estrato arbóreo: composición de especies, estructura de la vegetación e índices de diversidad, partiendo de parches de vegetación delineados a través de una clasificación orientada a objetos de imágenes multiespectrales. Se identificaron además otros atributos que distinguen los diferentes parches, tales como la naturaleza y tiempo después del disturbio. La hipótesis central es que es posible clasificar diferentes tipos de composición de especies y estructura del arbolado (hábitats o parches de vegetación) con base en datos multiespectrales, y que, con relativamente pocas variables de campo, es posible predecir su pertenencia a los mismos.

\section{MATERIALES Y MÉTODOS}

El estudio se desarrolló en 900 ha de selva mediana subperennifolia con distintos antecedentes de aprovechamiento, tales como agrícola bajo el sistema roza, tumba y quema (Ac: 8-23 años), silvícola selectivo (Fs: 43-53 años) y silvícola con afectación por incendios (Fc: 21-28 años) (Sánchez-Santos et al., 2015). El área pertenece al ejido Felipe Carrillo Puerto, Quintana Roo, México (18 $\left.53^{\prime} \mathrm{N}-88^{\circ} 14^{\prime} \mathrm{W}\right)$. El clima es cálido subhúmedo con lluvias en verano, con temperatura media anual de $26^{\circ} \mathrm{C}$ y precipitación pluvial anual promedio es de 1200mm (García, 1990). El paisaje es carsotectónico a menos de $20 \mathrm{~m}$ de altitud (Bautista, Palacio, Ortiz-Pérez, Batllori-Sampedro, \& Castillo-González, 2005). Se establecieron sistemáticamente 32 unidades de muestreo de una hectárea (UM) con algunas modificaciones para cubrir diferentes parches de vegetación (Fig. 1). Cada unidad se compone de 4
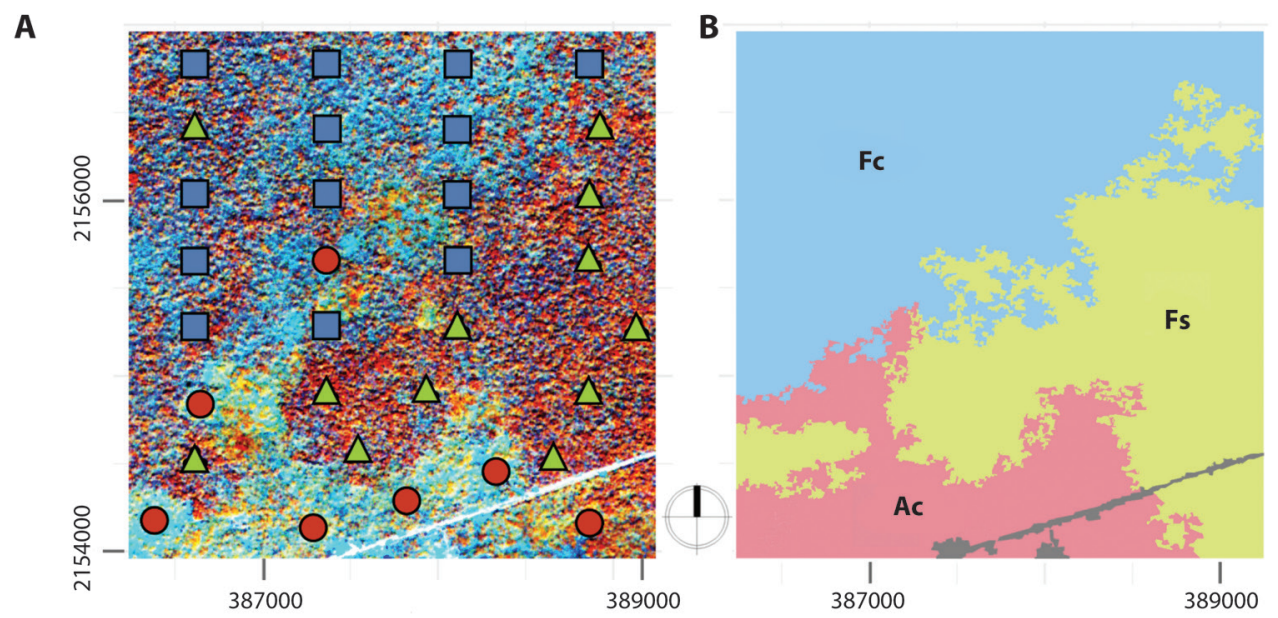

Fig. 1. (A) Unidades de muestreo sobre compuesto RGB, bandas infra roja, red edge y roja; las formas (círculo, triángulo y cuadrado) indica el parche predicho por el modelo (Ac: antecedente aprovechamiento agrícola, Fs: aprovechamiento silvícola y Fc: aprovechamiento silvícola con incendio forestal). (B) Clasificación multiespectral orientada a objetos. Mapas en UTM $16 \mathrm{~N}$.

Fig. 1. (A) Plots over rapidEye RGB composite: near infrared, red edge, red. Shape (circle, triangle, and square) indicate the patch predicted by the model (Ac: slash and burn agriculture, Fs: selective logging, Fc: selective logging and forest fire). (B) Object-oriented multiespectral classification. Maps in UTM 16N. 
subunidades circulares de $400 \mathrm{~m}^{2}$ arregladas en forma de Y invertida, similares a las del Inventario Nacional Forestal y de Suelos (INFyS) de México (CONAFOR, 2010).

De marzo a agosto 2013 se midieron los individuos vivos mayores a $7.5 \mathrm{~cm}$ de diámetro normal (dn a una altura de $1.30 \mathrm{~m}$ ). A cada individuo se le midió la altura total (at), el dn, y el diámetro de mayor de copa. Se identificó taxonómicamente y se corroboró la nomenclatura aceptada a nivel de familia, género y especie en la base de datos del Missouri Botanical Garden (Tropicos, 2013) y The Plant List (The Plant List, 2013).

Cálculo de variables de estructura de la vegetación: Para cada UM se calculó la media, la mediana y los percentiles 75 y 80 de las variables número de tallos (densidad), altura total, área basal, cobertura de copa y biomasa (calculada con las ecuaciones específicas ajustadas para árboles de $\mathrm{dn}<10 \mathrm{~cm}$ (Puc-Kauil, en prep.) para los individuos con $\mathrm{dn}>10 \mathrm{~cm}$ (Cairns, Olmsted, Granados, \& Argaez, 2003), lianas (Chave et al., 2003) y palmas (Delaney, Brown, \& Powell, 1999). Todas las variables fueron calculadas en conjunto, y separadas por el porte de los individuos ( $\mathrm{dn}<25 \mathrm{~cm}$, y dn $>25 \mathrm{~cm}$ ) (Román-Dañobeytia et al., 2014). También se estimó el error porcentual de las estimaciones.

Cálculo de diversidad y composición de especies: La importancia ecológica de las especies se expresó mediante el Valor de Importancia Relativa (VIR (\%) = 1/3 (Densidad Relativa + Área Basal Relativa + Frecuencia Relativa) (Mueller-Dombois \& Ellenberg, 1974). Para medir la diversidad estructural se crearon veinte categorías de diámetros, alturas y biomasa, y se calculó el índice de Shannon-Wiener $\mathrm{H}$ sobre sus frecuencias (Zhang \& Chen, 2015, Martínez-Sánchez, 2016). Para la diversidad taxonómica se calcularon los índices de Shannon-Wiener, Simpson 1/D, índice de Equidad, y número de especies por UM usando la función "diversity" del paquete "vegan" (Oksanen et al., 2018).
Adquisición y procesamiento de imágenes espectrales: Se gestionó la escena RapidEye 1648010 del 3 de marzo del 2013 con nivel $3 \mathrm{~A}$ de procesamiento. Ésta tiene una resolución espacial de $5 \mathrm{~m}$ en modo multiespectral y una resolución espectral de 5 bandas: azul (440 $510 \mathrm{~nm})$, verde $(520-590 \mathrm{~nm})$, roja (630 - 685 $\mathrm{nm})$, borde del rojo o "Red Edge" (690 - 730 $\mathrm{nm})$ e infrarroja cercana $(760-850 \mathrm{~nm})$. La presencia de la banda del borde del rojo es una característica única de las imágenes RapidEye, frecuentemente utilizadas para caracterizar tipos de vegetación, porque es especialmente sensible al estado de clorofila, hoja y estructura de copa. Los números digitales de 16 bits fueron transformados a reflectancia al tope de la atmósfera después de una transformación a radianza (Planet, 2016). Con este procedimiento se corrigieron las variaciones en la iluminación (Chuvieco-Salinero, 2008) asumiendo una atmósfera uniforme en toda la imagen.

De acuerdo con los antecedentes de uso de la tierra, se propuso segmentar el área en tres parches de vegetación, a partir de los datos multiespectrales, mediante una clasificación orientada a objetos con el software eCognition 9 (Trimble, 2014). Esta aproximación fue elegida sobre la clasificación convencional, porque el uso de objetos en vez de pixeles, reduce la variabilidad espectral dentro de cada clase, y minimiza el efecto sal y pimienta que se produce por la heterogeneidad individual de los pixeles (Liu \& Xia, 2010). El procedimiento requiere segmentar la imagen antes de la clasificación, para lo que se utilizó un algoritmo de segmentación multiresolución tipo "button-up", basado en la "Fractal Net Evolution Approach" (FNEA) (Baatz \& Schäpe, 2000).

La segmentación parte de objetos del tamaño de un pixel, y los combina secuencialmente sin traslapar, minimizando la heterogeneidad de los objetos resultantes hasta que excede un umbral definido por el parámetro de escala, que determinará el tamaño promedio de los objetos. El valor de heterogeneidad utilizado pondera las heterogeneidades de color y de forma, definidas con los parámetros "Shape" y "Compactness". En este caso, los parámetros fueron 
determinados empíricamente como sigue: factor de escala 3 , criterio de forma 0 , criterio de compactibilidad 0.6 , con pesos iguales a la banda verde y roja, y el doble a la banda infrarroja. Los objetos resultantes fueron clasificados en áreas sin vegetación (carretera) y con vegetación, y de esta última se identificaron 3 parches de vegetación.

\section{Comparación multivariada entre par-} ches de vegetación: Se aplicó una transformación logarítmica a los VIR (\%) de todas las especies para reducir la importancia de los valores muy elevados, y una estandarización de Wisconsin a las variables de estructura de la vegetación y de diversidad, dada la variedad de unidades y escalas de las matrices (Borcard, Gillet, \& Legendre, 2011). A partir de los valores transformados se generaron tres matrices de disimilitud basadas en el índice de Bray y Curtis (1957). Posteriormente se sometieron a un análisis de ordenamiento calculado con el método de escalamiento no métrico multidimensional (NMDS). Dicha técnica posiciona las UMs en un espacio dimensional reducido, con valores arbitrarios, pero representativos como indicadores relativos de disimilitud de la matriz original k-dimensional (Kruskal, 1964, Borcard et al., 2011). Ésta fue elegida sobre otras técnicas de ordenamiento porque no asume linealidad, puede partir de cualquier tipo de matriz de disimilitud (Legendre \& Legendre, 1998), y tiene precedente en percepción remota con estructura arbórea y composición específica (Wiggins, 2017; Hakkenberg et al., 2018a).

Se seleccionaron las UMs que se encuentran a más de $100 \mathrm{~m}$ de la división entre parches de vegetación, y se observó su variabilidad en los dos primeros ejes de variación del NMDS agrupadas por parche. Las diferencias en composición, estructura y diversidad fueron probadas con el análisis de varianza multivariado permutacional basado en matrices de disimilitud (PERMANOVA), usando la función "adonis" del paquete "vegan" (Anderson, 2005). Considerando que las diferencias en dispersión de los grupos pueden alterar la sensibilidad del PERMANOVA (Anderson \& Walsh, 2013), se realizó una prueba de homogeneidad de la dispersión multivariada usando la función "betadisper", que compara distancias no euclideanas entre los conglomerados y el centroide de la clase a la que pertenecen, reduciendo las distancias originales a coordenadas principales (Anderson, 2006). La prueba pareada de este análisis se corrió con la función "permutest" del paquete "vegan" (Oksanen, 2018).

Selección de variables significativas: Para estimar la probabilidad de pertenencia de las 32 UMs (utilizadas, y omitidas) a cada parche de vegetación, y determinar las variables significativamente definitorias en su delineamiento, se ajustó un modelo de regresión logística multinomial, en función de covariables de composición de especies, estructura de la vegetación e índices de diversidad, usando la función "multinom" del paquete "nnet" (Ripley \& Venables, 2016).

Se utilizaron procedimientos manuales y automatizados de selección de variables (función "stepwise" del paquete "MASS" (Venables \& Ripley, 2002), y se compararon el criterio de información Akaike (AIC) y la devianza para elegir los mejores modelos. Sus coeficientes y errores estándar se compararon con la función "compareCoefs" del paquete "car" (Fox et al., 2018). Posteriormente, se utilizó una prueba de razón de verosimilitud para modelos multinomiales para descartar que los modelos con mejor ajuste no presentaran diferencias estadísticas.

Finalmente, se utilizó el método no paramétrico de Kruskal-Wallis con la función "kruskal.test", y la prueba pareada de Wilcoxon con la función "pairwise.wilcox.test" para comparar una a una, las medianas de las covariables seleccionadas por el modelo entre parches de vegetación, y se analizó gráficamente su variación. Todos los análisis de datos se realizaron en R, v. 3.4.4 (R Core Team, 2016).

\section{RESULTADOS}

Delineamiento de parches de vegetación: La clasificación orientada a objetos delineó tres 
parches de vegetación (Fig. 1B). Es importante destacar que los límites entre los parches no necesariamente corresponden exactamente a los historiales de aprovechamiento, pero son un punto de partida para la caracterización de la vegetación arbórea. Las 32 UM fueron catalogadas de acuerdo con dicha clasificación, que agrupó la variabilidad espectral de la imagen: El parche Ac se distinguió por poseer los valores más altos de reflectancia en el espectro visible. La característica principal que distingue los parches $\mathrm{Fs}$ y $\mathrm{Fc}$, es que éste último presenta medias menores de reflectancia en la banda del infrarrojo.

Comparación multivariada entre parches de vegetación: Se consideraron 26 UMs en las ordenaciones para las matrices de composición, estructura y diversidad. Estas resultaron altamente representativas, con bajos valores de estrés $(0.14,0.07$ y 0.017$)$, y altas correlaciones lineales entre las distancias k-dimensionales originales y las bidimensionales (0.92, 0.98 y 0.99 respectivamente). En todos los casos, las UMs del mismo parche se observan semejantes (Fig. 2).

Se rechazó la Hipótesis nula $(\mathrm{H} 0)$ de igualdad entre las medias multivariadas (PERMANOVA, $\mathrm{P}<0.001)$. Las pruebas generales y pareadas de dispersión para las ordenaciones de composición y diversidad no rechazan la H0 (BETADISPER, $\mathrm{P}>0.01$ ), por lo que las diferencias encontradas en el PERMANOVA pueden ser atribuidas a diferencias en la localización de las medias multivariadas. En la matriz de estructura, sí se rechaza la dispersión multivariada con (BETADISPER, P > 0.01) para la prueba general y las pareadas Ac-Fs y Ac-Fc. Las diferencias detectadas en estos pares podrían ser explicadas por diferencias en la localización, dispersión o ambas, no así en la comparación entre los parches Fs y Fc (PERMUTEST, P > 0.01) cuyas diferencias son atribuibles a la posición de las medias.

Selección de variables significativas: El modelo logístico multinomial ajustado incluye como variables significativas el número de individuos por hectárea $(\mathrm{P}<0.001)$, el número de especies $(\mathrm{P}<0.01)$, y el primer eje de variación del análisis de ordenamiento NMDS ( $\mathrm{p}<0.01)$ para predecir la pertenencia de las UMs a un parche determinado. Este modelo se comparó con otros con ajustes promisorios (Tabla 1), y se eligió por su mejor ajuste sobre otros con menos variables, debido a que se encontraron diferencias significativas entre ellos (prueba de razón de verosimilitud, $\mathrm{P}<$ $0.05)$. Con el modelo seleccionado se generaron probabilidades de pertenencia de las 32 UMs a cada parche de vegetación. Todas ellas presentaron probabilidades de más del $99 \%$ hacia alguna clase, y se presentaron dos UMs espectralmente clasificadas como Fc y predichas como Ac, y Fs (Fig. 1A).

Caracterización de parches de vegetación: El parche de vegetación Ac es dominado por Bursera simaruba (L.), seguida por Piscidia piscipula (L.) y Vitex gaumeri (Greenm.) (Tabla 2), el primer eje del NMDS sobre la composición específica mostró diferencias significativas entre este parche y los otros dos (Wilcoxon, $\mathrm{P}<0.001$ ). La familia Leguminosae es la más representativa en número de individuos (207 tallos/ha), y número especies (12) para este parche, seguida por la familia Polygonaceae con 4 especies. Sus promedios

TABLA 1

Métricas de bondad de ajuste de los modelos con menores valores de AIC

TABLE 1

Goodness of fit metrics of the models with less AIC values

\begin{tabular}{|c|c|c|c|c|c|}
\hline & & \multicolumn{2}{|c|}{ Modelo } & Devianza & AIC \\
\hline a) & $\begin{array}{c}\mathrm{R}+ \\
*\end{array}$ & $\begin{array}{l}\mathrm{D}+ \\
* * *\end{array}$ & $\begin{array}{c}\mathrm{C} 1 \\
*\end{array}$ & 0.09 & 16.09 \\
\hline b) & $\begin{array}{c}\mathrm{R}+ \\
*\end{array}$ & $\begin{array}{c}\mathrm{D}+ \\
*\end{array}$ & $\begin{array}{c}\log 1 \mathrm{p}(\mathrm{C} 1) \\
* * *\end{array}$ & 0.06 & 16.09 \\
\hline c) & & $\begin{array}{l}\mathrm{D}+ \\
* * *\end{array}$ & $\begin{array}{l}\mathrm{C} 1 \\
* *\end{array}$ & 6.7 & 18.7 \\
\hline d) & & $\begin{array}{l}\mathrm{D}+ \\
* * *\end{array}$ & $\begin{array}{c}\log 1 \mathrm{p}(\mathrm{C} 1) \\
* *\end{array}$ & 6.37 & 18.4 \\
\hline
\end{tabular}

$\mathrm{R}=$ número de especies, $\mathrm{D}=$ densidad (tallos/ha), $\mathrm{Cl}$ =primer eje del nMDS para la matriz de composición. La significancia se indica debajo de cada covariable: $\mathrm{P}<$ $0.0001=* * *, \mathrm{P}<0.001=* *,, \mathrm{P}<0.01=*$. 


\section{Composición}
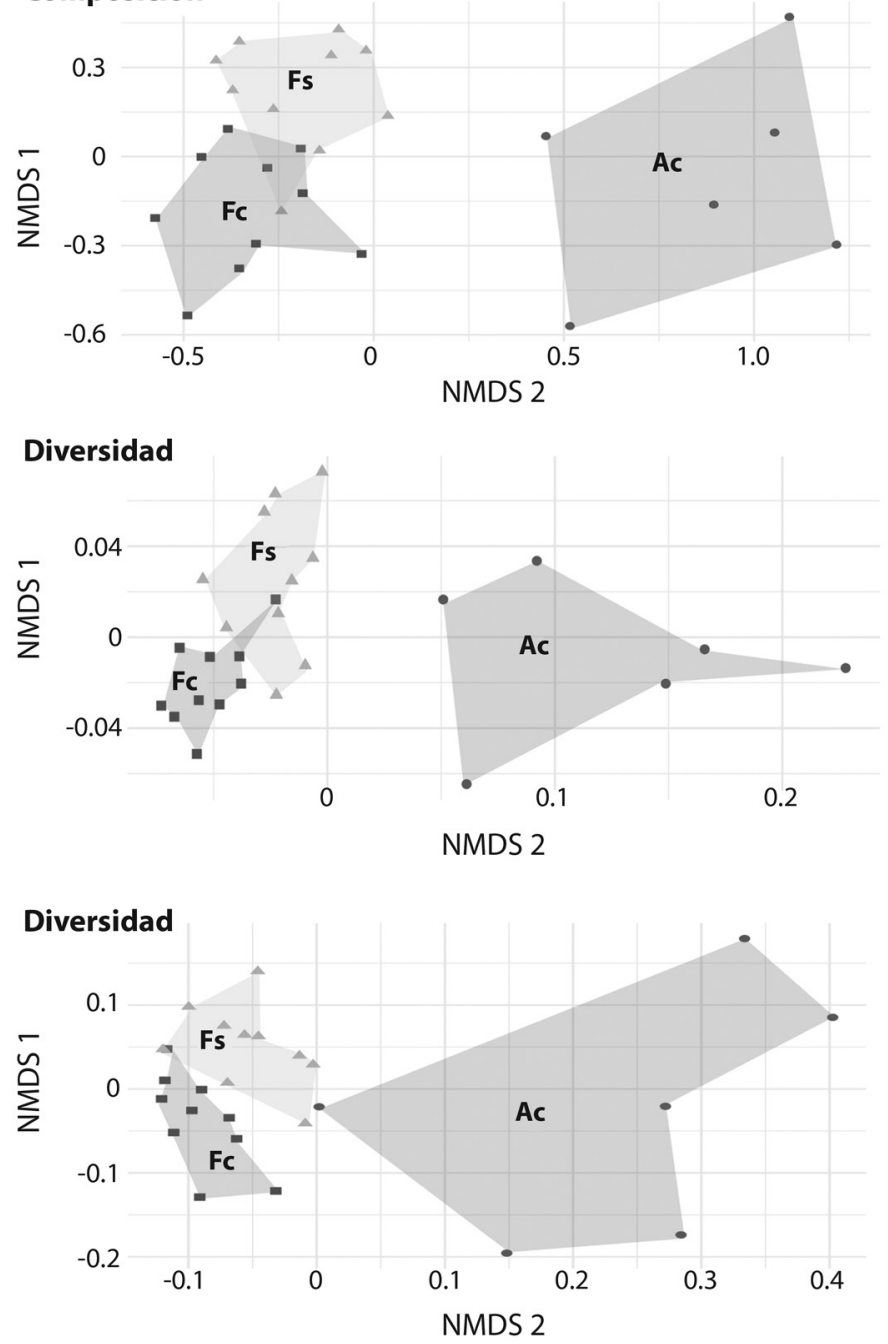

Fig. 2. Unidades de muestreo en el espacio NMDS definido por matrices de disimilitud. Los polígonos envolventes representan el parche de vegetación al que pertenecen. Ac: antecedente aprovechamiento agrícola, Fs: aprovechamiento silvícola y Fc: aprovechamiento silvícola con incendio forestal.

Fig. 2. Plots in the NMDS space of the dissimilarity matrices. Remotely sensed patches are indicated by polygon hulls. Ac: slash and burn agriculture, Fs: selective logging, Fc: selective logging and forest fire.

de número de especies e índices de ShannonWiener sobre diámetros y alturas, son menores y significativamente distintos a los otros dos (Kruskal-Wallis, $\mathrm{P}<0.001$, Wilcoxon, $\mathrm{P}<$ 0.01) (Tabla 3). El índice de Shannon-Wiener es significativamente menor que en el Fc (Wilcoxon, $\mathrm{P}<0.01)$, pero no menor que el del Fs (Wilcoxon, $\mathrm{P}<0.05$ ) (Fig. 3).
La estructura del parche Ac se caracteriza por tener los menores promedios de altura, área basal individual al percentil 80 (P80), y de densidad, biomasa y área basal por hectárea, para todos los individuos, y para los de dn $>$ $25 \mathrm{~cm}$ (Kruskal-Wallis, $\mathrm{P}<0.001$, Wilcoxon, $\mathrm{P}<0.01$ ) (Tabla 2). No obstante, los promedios individuales de biomasa al P80 no son 
TABLA 2

Valores estructurales de las diez especies arbóreas con mayor Valor de Importancia Relativa (VIR)

porcentual por parche de vegetación

TABLE 2

Structural values of the 10 most important tree species according to the porcentual Relative Importance Value (VIR) by remotely sensed vegetation patches

\begin{tabular}{|c|c|c|c|c|c|c|c|c|c|c|c|c|c|}
\hline \multirow{2}{*}{ Fam } & \multirow{2}{*}{ Especie } & \multicolumn{4}{|c|}{ Ac } & \multicolumn{4}{|c|}{ Fs } & \multicolumn{4}{|c|}{$\mathrm{Fc}$} \\
\hline & & AR & DR & FR & VIR (R) & $\mathrm{AR}$ & DR & FR & VIR (R) & $\mathrm{AR}$ & DR & FR & $\operatorname{VIR}(\mathrm{R})$ \\
\hline $\mathrm{BU}$ & Bursera simaruba & 12 & 21 & 9 & $14(1)$ & 5 & 3 & 4 & $4(5)$ & 12 & 6 & 4 & $7(2)$ \\
\hline EU & Gymnanthes lucida & - & - & - & - & 10 & 31 & 6 & $16(1)$ & 8 & 22 & 5 & $12(1)$ \\
\hline LE & Piscidia piscipula & 15 & 14 & 8 & $12(2)$ & - & - & - & - & - & - & - & - \\
\hline $\mathrm{SO}$ & Manilkara zapota & 8 & 1 & 2 & $4(6)$ & 20 & 9 & 6 & $12(2)$ & 5 & 3 & 3 & $4(6)$ \\
\hline LA & Vitex gaumeri & 17 & 8 & 7 & $11(3)$ & 8 & 2 & 3 & $4(4)$ & - & - & - & - \\
\hline MA & Pseudobombax ellipticum & - & - & - & - & 13 & 3 & 4 & $7(3)$ & 11 & 4 & 4 & $6(3)$ \\
\hline LE & Lysiloma latisiliquum & 8 & 6 & 4 & $6(4)$ & - & - & - & - & - & - & - & - \\
\hline LE & Caesalpinia gaumeri & 4 & 7 & 3 & $5(5)$ & 4 & 3 & 3 & $3(6)$ & 8 & 5 & 4 & $6(4)$ \\
\hline SI & Thouinia paucidentata & - & - & - & - & - & - & - & - & 4 & 5 & 4 & $4(5)$ \\
\hline $\mathrm{PO}$ & Coccoloba spicata & 2 & 4 & 4 & $3(7)$ & 3 & 2 & 3 & $3(8)$ & 3 & 2 & 2 & $2(10)$ \\
\hline $\mathrm{SO}$ & Pouteria reticulata & - & - & - & - & 2 & 4 & 3 & $3(7)$ & - & - & - & - \\
\hline $\mathrm{AN}$ & Metopium brownei & - & - & - & - & - & - & - & - & 5 & 2 & 3 & $3(7)$ \\
\hline MA & Luehea speciosa & 2 & 3 & 4 & $3(8)$ & - & - & - & - & - & - & - & - \\
\hline PU & Drypetes lateriflora & - & - & - & - & - & - & - & - & 2 & 4 & 3 & $3(8)$ \\
\hline $\mathrm{AN}$ & Spondias radlkoferi & 2 & 2 & 4 & $3(9)$ & - & - & - & - & - & - & - & - \\
\hline SI & Exothea paniculata & - & - & - & - & 2 & 3 & 4 & $3(9)$ & - & - & - & - \\
\hline LE & Lonchocarpus xuul & - & - & - & - & - & - & - & - & 2 & 4 & 3 & $3(9)$ \\
\hline MO & Ficus sp. & 3 & 2 & 3 & $3(10)$ & - & - & - & - & - & - & - & - \\
\hline ME & Trichilia hirta & - & - & - & - & 1 & 3 & 3 & $3(10)$ & - & - & - & - \\
\hline
\end{tabular}

Ac: parche de vegetación con antecedente aprovechamiento agrícola, Fs: aprovechamiento silvícola y Fc: aprovechamiento silvícola con incendio forestal, AR: área basal relativa; DR: densidad relativa; FR: frecuencia relativa; VIR: valor de importancia relativa, R: posición con respecto al VIR. BU: Burseraceae, EU: Euphorbiaceae, LE: Leguminosae, SO: Sapotaceae, LA: Lamiaceae, MA: Malvaceae, SI: Sapindaceae, Po: Polygonaceae, AN: Anacardiaceae, PU: Putranjivaceae, MO: Moraceae, ME: Meliaceae.

significativamente distintos (Wilcoxon, $\mathrm{P}>$ 0.05) (Fig. 3). Se distinguen 19 individuos aislados de más de $15 \mathrm{~m}$ de altura, principalmente de las especies: Manilkara zapota (L.), V. gaumeri, y P. piscipula.

El parche Fs es dominado por Gymnanthes lucida (Sw.), seguida por M. zapota y Pseudobombax ellipticum (Kunth) (Tabla 1). La familia Euphorbiaceae tiene mayor densidad (303 tallos/ha), la Sapotaceae mayor área basal, y la Leguminosae más especies (16). Los promedios de riqueza taxonómica e índices de Shannon-Wiener sobre especies, diámetros y alturas de este parche y el Fc, son mayores a los del Ac (Kruskal-Wallis, $\mathrm{p}<0.001$, Wilcoxon, $\mathrm{P}$ $<0.01$ ) (Fig. 3) pero tanto las medias de riqueza, como del índice de Shannon-Wiener sobre especies, son superiores en el parche Fc que en el Fs (Wilcoxon, $\mathrm{P}<0.01$ ) (Fig. 3). Este último es dominado por G. lucida, seguida por $B$. simaruba y $P$. ellipticum (Tabla 1). La familia dominante es Euphorbiaceae (287 tallos/ha), seguida por la familia Leguminosae. El segundo eje del NMDS sobre la composición específica mostró diferencias significativas entre los parches de vegetación Fc y Fs (Wilcoxon, $\mathrm{P}<0.01)$. 
TABLA 3

Métricas estructurales e índices de diversidad promedio entre parches de vegetación

TABLE 3

Mean structural metrics and diversity indexes by remotely sensed vegetation patches

\begin{tabular}{llcccc} 
& & Ac* & Fs* & Fc* & SMIC* \\
Percentil 80 & $\mathrm{AT}(\mathrm{m})$ & $9.3 \pm 5.1$ & $14.2 \pm 1.5$ & $13.8 \pm 2$ & $12.9 \pm 6$ \\
individual & $\mathrm{AB}\left(\mathrm{m}^{2}\right)$ & $0.016 \pm 10.2$ & $0.039 \pm 6.9$ & $0.034 \pm 7.7$ & $0.032 \pm 12.6$ \\
& $\mathrm{BM}(\mathrm{kg})$ & $164 \pm 18.2$ & $261.7 \pm 9.1$ & $215.5 \pm 6.7$ & $221.3 \pm 11.4$ \\
Métricas & $\mathrm{n}(\mathrm{tallos} / \mathrm{ha})$ & $669 \pm 16$ & $988 \pm 4$ & $1306 \pm 2.5$ & $1074 \pm 9$ \\
por ha & $\mathrm{AB}\left(\mathrm{m}^{2} / \mathrm{ha}\right)$ & $10.7 \pm 19$ & $30.6 \pm 6$ & $32.6 \pm 3.6$ & $27 \pm 12.5$ \\
& $\mathrm{BM}(\mathrm{Mg} / \mathrm{ha})$ & $71 \pm 17$ & $201 \pm 7$ & $203 \pm 3.8$ & $177 \pm 12$ \\
Métricas & $\mathrm{n}(\mathrm{tallos} / \mathrm{ha})$ & $32 \pm 35$ & $160 \pm 5.2$ & $163 \pm 10$ & $134 \pm 16$ \\
por ha & $\mathrm{AB}\left(\mathrm{m}^{2} / \mathrm{ha}\right)$ & $3.77 \pm 33$ & $18.9 \pm 9$ & $15.9 \pm 8.4$ & $14.1 \pm 16$ \\
dap $>$ 25 cm & $\mathrm{BM}(\mathrm{Mg} / \mathrm{ha})$ & $22.98 \pm 35$ & $136 \pm 11.2$ & $102 \pm 8.2$ & $96 \pm 19$ \\
Índices diversidad & \# (especies (familias)) & $24(15) \pm 10$ & $37(20) \pm 3.6$ & $45(22) \pm 3.8$ & $37 \pm 7.9$ \\
taxonómica & $\mathrm{H}^{\prime} \mathrm{sp}$ & $2.5 \pm 4.3$ & $2.8 \pm 2.8$ & $3.1 \pm 2$ & $2.89 \pm 3.7$ \\
Índices diversidad & $\mathrm{H}^{\prime} \mathrm{DN}$ & $2.6 \pm 3.3$ & $2.9 \pm 0.5$ & $2.9 \pm 0.2$ & $2.9 \pm 2.3$ \\
estructural & $\mathrm{H}^{\prime} \mathrm{AT}$ & $2.1 \pm 5.1$ & $2.7 \pm 0.8$ & $2.7 \pm 0.5$ & $2.6 \pm 4$ \\
& $\mathrm{H}^{\prime} \mathrm{DN}$ & $2.5 \pm 3.9$ & $2.9 \pm 0.5$ & $2.9 \pm 0.5$ & $2.9 \pm 2.8$ \\
\hline
\end{tabular}

Ac: parche de vegetación con antecedente aprovechamiento agrícola, Fs: aprovechamiento silvícola selectivo y Fc: aprovechamiento silvícola selectivo con incendio forestal, SMIC: Todas las unidades de muestreo del Sitio de Monitoreo Intensivo de Carbono, $*=$ Promedio \pm error estándar (\%). BM (biomasa), DN (diámetro normal), AT (altura), n (densidad), $\mathrm{AB}$ (área basal), H' (Índice Shannon-Wiener), S (Índice Simpson).

En cuanto a su estructura, parche Fs tiene mayores medias de altura de dosel, área basal y biomasa individual al percentil 80 , y área basal, y biomasa por hectárea que el Fc pero las diferencias no son significativas (Wilcoxon, $\mathrm{P}>0.05$ ) (Tabla 2). La densidad de individuos por hectárea de los individuos mayores a $25 \mathrm{~cm}$ tampoco resultó significativamente distinta a la del Fc (Wilcoxon, $\mathrm{P}>0.05$ ) (Tabla 2). Pero al considerar todos los individuos se evidenció que la media del $\mathrm{Fc}$ es significativamente mayor (Wilcoxon, $\mathrm{P}<0.001$ ) (Fig. 3). Ambos parches de vegetación tienen más de 15 veces más tallos mayores a $25 \mathrm{~cm}$ que el parche Ac.

\section{DISCUSIÓN}

La mayor reflectancia promedio del parche Ac en el espectro visible, es consistente con su mayor abundancia relativa de especies caducifolias, y por tanto, menor área foliar expuesta en una imagen tomada al inicio de la estación seca. Esto es explicable, ya que el tejido foliar suele presentar bajas reflectancias en este rango debido a la fuerte absorción de la clorofila (Knipling, 1970), y si bien las especies caducifolias persisten a lo largo de la sucesión (Egler, 1954), se ha observado mayor abundancia y diversidad de éstas en etapas sucesionales tempranas (Finegan, 1996).

La diferencia en promedios espectrales en la banda del infrarrojo entre los parches Fs y $\mathrm{Fc}$, se debe a que las ondas de luz correspondientes a esta región del espectro electromagnético, son típicamente reflejadas para evitar el calentamiento del tejido foliar (Knipling, 1970), por lo que se infiere que la mayor reflectancia del parche Fs se debe a mayor superficie de área foliar expuesta, o bien a contar con vegetación más vigorosa. Dichas características son evidentes en el compuesto falso color (Fig. 1) en que los rojos más intensos representan altas reflectancias en la banda infraroja. Los tonos celestes en el tercio inferior de la imagen 

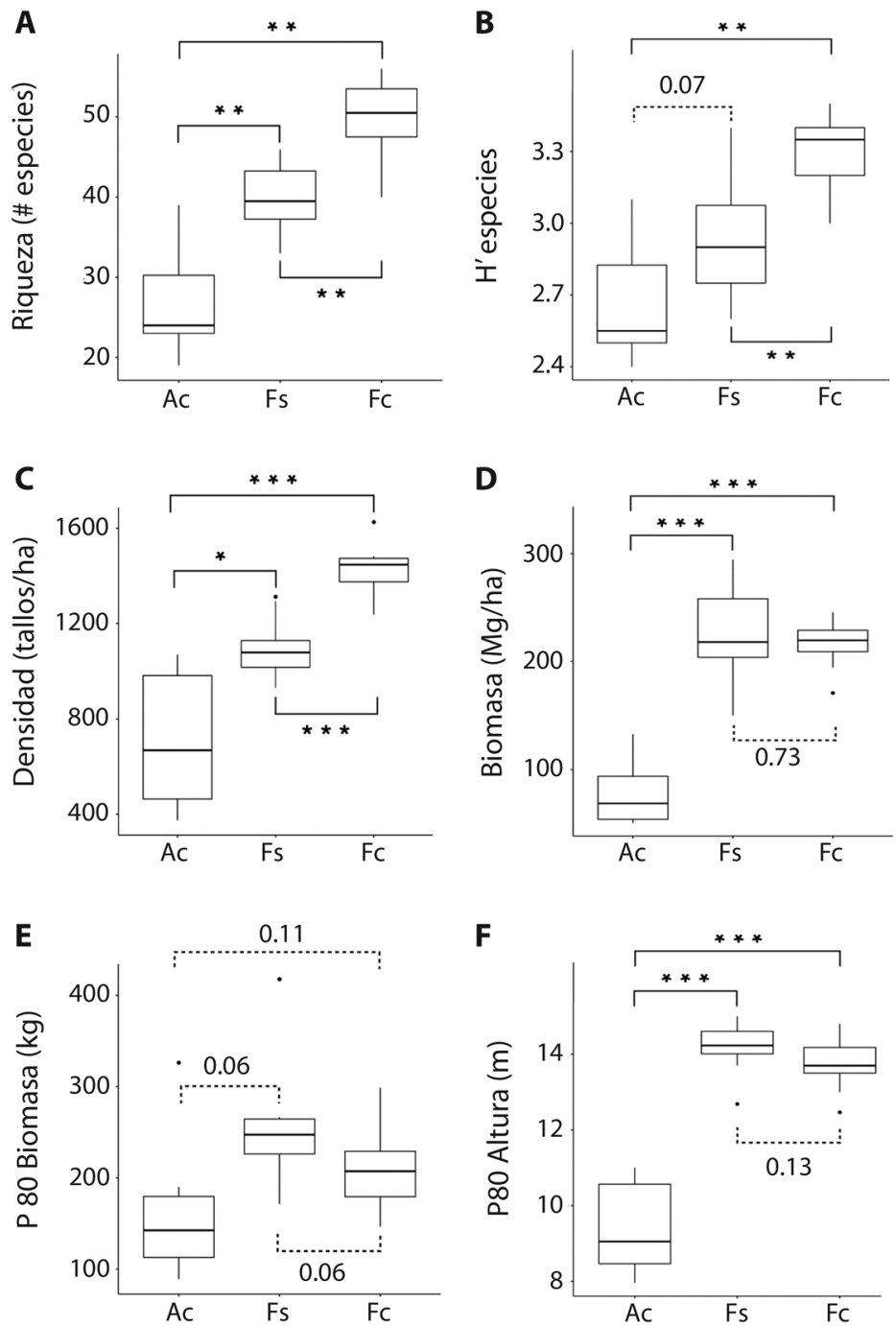

Fig. 3. Comparación univariada de atributos entre parches de vegetación. Ac: antecedente aprovechamiento agrícola, Fs: aprovechamiento silvícola y Fc: aprovechamiento silvícola con incendio forestal. Significancia de la prueba de Wilcoxon: $\mathrm{P}<0.0001=* * *, \mathrm{P}<0.001=* *,, \mathrm{P}<0.01=*$.

Fig. 3. Univariate attribute comparison between remotely sensed patches. Ac: slash and burn agriculture, Fs: selective logging, Fc: selective logging and forest fire. Significance for the pairwise Wilcoxon: $\mathrm{P}<0.0001=* * *, \mathrm{P}<0.001=* *$,, $\mathrm{P}<$ $0.01=*$.

tienen mayor reflectancia en el canal rojo del espectro visible. Esta clasificación se relacionó eficientemente con los datos de estructura de la vegetación, composición de especies e índices de diversidad.

El modelo multinomial permitió identificar a la riqueza de especies, el número de individuos mayores a $7.5 \mathrm{~cm}$ por hectárea, y la composición de especies como variables clave en la diferenciación de los parches homogéneos de vegetación, confirmando la hipótesis de que la clasificación espectral orientada a objetos es consistente con la estructura de la vegetación y la composición de especies, y que conociendo las tres variables anteriores es posible predecir el parche de vegetación al que cada UM 
pertenece. Los valores ajustados no mostraron UMs transicionales; es decir, en todos los casos, la probabilidad de pertenencia a algún parche fue superior al $90 \%$. Esta situación es coherente con que las diferencias entre los parches no se deben a gradientes ambientales sino a perturbaciones antropogénicas o naturales de límites fijos.

Por otra parte, existe la posibilidad de que los dos errores en la clasificación registrados representen verdaderamente el parche al que fueron asignados de acuerdo a sus atributos de campo, pero que el parámetro de escala utilizado en la clasificación orientada a objetos haya absorbido dicha variabilidad en objetos de mayor talla (Liu \& Xia, 2010). Esta metodología puede utilizarse en otros paisajes con pocas clases a distinguir, ya que los modelos multinomiales fueron específicamente diseñados para problemas de este tipo.

La evaluación y seguimiento de la composición de especies arbóreas mediante NMDS es más útil en la evaluación de los efectos del manejo forestal en la diversidad, que la riqueza de especies (Imai et al., 2014). Sus primeros ejes de variación se han relacionado con sensores remotos para crear modelos continuos de composición (Hakkenberg et al., 2018a). Esta técnica también se ha usado sobre los VIR de las especies por clases diamétricas en selvas medianas subperennifolias para evaluar efectos del aprovechamiento (Gutiérrez-Granados et al., 2011). Además, la composición de especies se ha relacionado con la sucesión desde gremios ecológicos (Sánchez-Sánchez et al., 2007; Granados-Victorino et al., 2017), y como indicador de degradación (Imai et al., 2014). Es influenciada por suelo, fertilidad, autocorrelación espacial (Dupuy et al., 2012), gradientes de humedad y edad después de un disturbio (Letcher et al., 2015).

La riqueza de especies es un atributo común en los estudios de modelado espacial con sensores remotos (Gallardo-Cruz et al., 2018; Hakkenberg, Gillet, \& Legendre, 2018b), y es considerada una variable relevante para distinguir parches de vegetación sucesionalmente distintos (Granados-Victorino et al.,
2017). De hecho, en el sito de estudio, ya se ha estudiado la riqueza en relación con la biomasa aérea (Hernández-Stefanoni et al., 2014).

La densidad de individuos es relevante porque es uno de los dos atributos que se evalúan para definir si una selva es secundaria o madura (Román-Dañobeytia et al., 2014), y se ha utilizado en conjunto con los niveles de biomasa, para estudiar la degradación de ecosistemas tropicales (Korom et al., 2016). Ecológicamente es relevante debido a su relación con la cantidad de luz que penetra el dosel, y las implicaciones sobre la funcionalidad del ecosistema sucesional (Finegan, 1996). También ha sido modelado a escalas globales con información espectral, topográfica y ambiental (Crowther et al., 2015).

Si bien las variables anteriores fueron las que presentaron las diferencias más evidentes entre los parches de vegetación, también se encontraron algunas diferencias en abundancia y diversidad a nivel de familia, índice de Shannon-Wiener, y otras métricas de altura, área basal o biomasa, coherentes con su tiempo desde el disturbio y su antecedente de uso (Tabla 1).

El parche Ac se encuentra entre las etapas sucesionales temprana y media, por su tiempo de abandono después de aprovechamiento agrícola (Gómez-Pompa, 1987). Ya se ha registrado en otras selvas aledañas con perturbaciones, la dominancia en VIR de $B$. simaruba (Carreón-Santos \& Valdez-Hernández, 2014). Este parche tiene también la mayor abundancia relativa de individuos de la familia Leguminosae, probablemente debido a que los árboles capaces de fijar nitrógeno son favorecidos en etapas tempranas después de un disturbio (Batterman et al., 2013). Su relación con la disponibilidad de agua es clara bajo los $1500 \mathrm{~mm}$ de precipitación anual, al punto que llega a tener el doble de área basal en selvas secundarias estacionales, que en perennes (Gei et al., 2018).

Este parche también tiene la menor diversidad y biomasa, atributos esperables dado su antecedente agrícola y menor tiempo desde el disturbio (Granados-Victorino et al., 2017; Hernández-Stefanoni et al., 2010). Su biomasa 
total promedio (56 Mg/ha) lo ubica en la categoría estructural de 16-29 años de recuperación (Urquiza-Haas et al., 2007), mientras el área basal y número de individuos lo define como vegetación secundaria (Román-Dañobeytia et al., 2014). La presencia de individuos aislados de porte grande, de especies características de selvas maduras como M. zapota y $V$. gaumeri (Sánchez-Sánchez et al., 2007), es común en selvas secundarias, ya sea por ser dejados para sombra, o por la dificultad para talarlos (Guevara-Sada, Laborde-Dovalí, \& Sánchez-Ríos, 2005).

La semejanza entre los parches de vegetación Fs y Fc, está relacionada con el hecho de que ambos tuvieron un aprovechamiento silvícola extensivo (Flashenberg \& Galleti, 1999), sin embargo, el parche Fc sufrió disturbios hace 21-28 años (incendio forestal) (Sánchez-Santos et al., 2015). En ambos parches de vegetación la familia con mayor abundancia relativa es la Euphorbiaceae, pero el parche Fs tiene mayor valor de abundancia relativa, situación coherente con su mayor tiempo desde el disturbio (Prieto et al., 2017), pero es opuesta a la teoría de la disminución de la codominancia con el incremento del tiempo desde el disturbio que han respaldado otros trabajos (Dupuy et al., 2012). La mayor diversidad específica del parche Fc, es coherente con otros casos en que se ha visto que sitios con disturbios ligeros presentan mayores valores de riqueza de especies (Carreño-Rocabado, Peña-Claros, Bongers, Licona, \& Poorter, 2012, Carreón-Santos \& Valdez-Hernández, 2014).

El parche Fs tiene menor densidad de tallos que el parche $\mathrm{Fc}$, pero mayores promedios de biomasa, área basal y alturas individuales. Si consideramos que éste último sufrió la perturbación más reciente, se explica la disminución de la densidad de tallos por su mayor tiempo al disturbio (Dupuy et al., 2012; Granados-Victorino et al., 2017), así mismo, la correlación negativa entre densidad y las otras variables, ha sido reportada (Urquiza-Haas et al., 2007). Considerando lo anterior, pareciera incoherente que el parche Ac (disturbio más reciente) tenga menor densidad, no obstante, es posible explicar este fenómeno al observar que las mayores densidades de individuos de este parche, tienen diámetros menores a $7.5 \mathrm{~cm}, \mathrm{y}$ no se contabilizaron en esta métrica.

Los parches Fs y Fc tienen individuos con dn $>25 \mathrm{~cm}$ suficientes como para ser consideradas selvas maduras (Román-Dañobeytia et al., 2014), aunque su media de área basal por hectárea aún está por debajo del límite de los $20 \mathrm{~m}^{2} /$ ha. La biomasa promedio total de ambos es alta y semejante, lo que los ubicaría en la categoría de más de 50 años, según los promedios regionales (Urquiza-Haas et al., 2007), y concuerda con los cálculos reportados para el sitio (Hernández-Stefanoni, 2014).

En general, es notable que las incertidumbres en las estimaciones de los promedios por hectárea de biomasa, área basal y número de individuos disminuyeron al analizar por separado cada parche debido a la agrupación de la variabilidad en los datos de campo (McRoberts, Tomppo, \& Czaplewski, 2015) a partir de la agrupación de la variabilidad espectral con una clasificación orientada a objetos. Por lo tanto, se confirman las hipótesis planteadas, concluyendo que la clasificación orientada a objetos con información espectral permitió un delineamiento de parches coherente con la estructura de la vegetación y la composición de especies, y que con relativamente pocas variables, identificadas como significativas por el modelo multinomial (primer eje de la composición específica, riqueza de especies, y densidad de individuos), es posible predecir la pertenencia de cada UM a los parches de vegetación clasificados.

\section{AGRADECIMIENTOS}

Esta investigación fue financiada por el Programa de Paisajes Sustentables de la Agencia para el Desarrollo Internacional de Estados Unidos de América, a través de la Oficina de Programas Internacionales del Servicio Forestal del Departamento de Agricultura, y El Northern Research Station. Se agradece a la Gerencia de Sistema Nacional de Monitoreo Forestal de la Comisión Nacional Forestal por 
las imágenes RapidEye, y al ejido de Felipe Carrillo Puerto y Uyoolche A. C. por el acceso y facilidades brindadas en el desarrollo del proyecto, especialmente a Filiberto Yam Buenfil y José Arreola Palacios. Así mismo, APOF agradece al Consejo Nacional de Ciencia y Tecnología la beca para realizar los estudios de Maestría.

\section{RESUMEN}

Tree density, species richness and composition define remotely-sensed vegetation patches in a subperennial forest. Recientemente se ha abordado la delimitación de parches de vegetación a través de clasificaciones orientadas a objetos con datos multiespectrales. En esta investigación se presenta una propuesta de caracterización de selvas secundarias basada en matrices de semejanza de composición específica, estructura de la vegetación arbórea e índices de diversidad, partiendo de una clasificación orientada a objetos de imágenes multiespectrales. El área de estudio corresponde a una selva mediana subperennifolia con antecedente de aprovechamiento agrícola bajo el sistema de roza tumba y quema (Ac: hace 8-23 años), aprovechamiento forestal selectivo (Fs: hace 43-53 años), y aprovechamiento forestal selectivo e incendio forestal (Fc: hace 21-28 años). Se delinearon tres parches de vegetación de acuerdo al historial de uso, y se analizaron las diferencias entre las medias de las métricas de campo de cada parche con un análisis de varianza multivariado permutacional $(\mathrm{P}<0.001)$. Con un modelo logístico multinomial se identificaron, de entre más de 20 covariables, la riqueza de especies, densidad de tallos por hectárea, y el primer eje de variación del ordenamiento NMDS de la composición específica, como los atributos de la vegetación más distintivos. El parche Fc, presenta los mayores valores en diversidad de Shannon-Wiener, riqueza de especies, y densidad de tallos mayores a $7.5 \mathrm{~cm}$ de diámetro. El Fs tiene los mayores promedios de biomasa, área basal y altura individual, y el Ac tiene los menores valores de todas las métricas anteriores. Las especies de mayor Índice de Valor de Importancia fueron: Ac; Bursera simaruba y Piscidia piscipula, Fs: Gymnanthes lucida y Manilkara zapota, Fc: G. lucida y B. simaruba.

Palabras clave: RapidEye; segmentación de imágenes; Índice de valor de importancia; permanova; modelo multinomial; bosque tropical; caracterización de hábitat.

\section{REFERENCIAS}

Anderson, M. (2005). Permutational multivariate analysis of variance (Tech. Rep.). Auckland, NZ: University of Auckland, Department of Statistics.
Anderson, M. (2006). Distance-based tests for homogeneity of multivariate dispersions. Biometrics, 62, 245-253.

Anderson, M., \& Walsh, D. (2013). PERMANOVA, ANOSIM, and the mantel test in the face of heterogeneous dispersions: What null hypothesis are you testing? Ecological Monographs, 83(4), 557-574.

Asaad, I., Lundquist, C. J., Erdmann, M. V., \& Costello, M. J. (2016). Ecological criteria to identify areas for biodiversity conservation. Biological Conservation, 213, 309-316.

Baatz, M., \& Schäpe, A. (2000). Multiresolution segmentation: an optimization approach for high quality multiscale image segmentation. In J. Strobl, T. Blaschke, \& G. Griesbner (Eds.), Angewandte Geographische Informations Verarbeitung XII (pp. 12-23). Germany: Wichmann Verlag.

Báez-Vargas, A. M., Esparza-Olguín, L., Martínez-Romero, E., Ochoa, S., Ramírez-Marcial, N., \& GonzálezValdivia, N. A. (2017). Efecto del manejo sobre la diversidad de árboles en vegetación secundaria en la Reserva de la Biosfera de Calakmul, Campeche, México. Revista de Biología Tropical, 65, 41-53.

Batterman, S. A., Hedin, L. O., Breugel, M. V., Ransijn, J., Craven, D. J., \& Hall, J. S. (2013). Key role of symbiotic dinitrogen fixation in tropical forest secondary succession. Nature, 502, 224-227.

Bautista, F., Palacio, G., Ortiz-Pérez, M., Batllori-Sampedro, E., \& Castillo-González, M. (2005). El origen y el manejo maya de las geoformas, suelos y aguas en la Península de Yucatán. En F. Bautista \& G. Palacio (Eds.), Caracterización y manejo de los suelos de la Península de Yucatán: Implicaciones agropecuarias, forestales y ambientales (pp. 21-32). Campeche, México: Universidad Autónoma de Campeche, Universidad Autónoma de Yucatán, Instituto Nacional de Ecología.

Borcard, D., Gillet, G. F., \& Legendre, P. (2011). Numerical ecology with $R$. New York, USA: Springer.

Bray, J. R., \& Curtis, J. T. (1957) An ordination of the upland forest communities of Southern Wisconsin. Ecological Monographs, 27, 325-349.

Cairns, M., Olmsted, I., Granados, J., \& Argaez, J. (2003). Composition and aboveground tree biomass of a dry semi-evergreen forest on Mexico's Yucatan Peninsula. Forest Ecology and Management, 186, 125-132.

Carreño-Rocabado, G., Peña-Claros, M., Bongers, F., Licona, J. C., \& Poorter, L. (2012). Effects of disturbance intensity on species and functional diversity in a tropical forest. Journal of Ecology, 100, 1453-1463.

CBD (Convention on Biological Diversity). (2010). Strategic Plan for Biodiversity 2011-2020 and the Aichi 
Targets. Canadá: United Nations. Recuperado de https://www.cbd.int/sp/default.shtml

Carreón-Santos, R. J., \& Valdez-Hernández, J. I. (2014). Estructura y diversidad arbórea de vegetación secundaria derivada de una selva mediana subperennifolia en Quintana Roo. Revista Chapingo Serie Ciencias Forestales y del Ambiente, 20, 119-130.

Chan-Dzul, A. M. (2010). Diversidad florística y funcional a través de una cronosecuencia de la selva mediana subperennifolia en la zona de influencia de la Reserva de la Biosfera Calakmul, Campeche, México (Tésis de Maestría). Centro Agronómico Tropical de Investigación y Enseñanza, Costa Rica.

Chave, J., Condit, R., Lao, S., Caspersen, J. P., Foster, R. B., \& Hubell, S. P. (2003). Spatial and temporal variation of biomass in a tropical forest: results from a large census plot in Panama. Journal of Ecology, 91, 240-252.

Chazdon, R. L., Broadbent, E. N., Rozendaal, D. M. A., Bongers, F., María, A., Zambrano, A., . . . Steininger, M. K. (2016). Carbon sequestration potential of second-growth forest regeneration in the Latin American tropics. Science Advances, 2(5), e1501639.

Chuvieco-Salinero, E. (2008). Teledetección ambiental (3ra ed.). Barcelona, España: Ariel Ciencias.

CONABIO (Comisión Nacional para el Conocimiento y Uso de la Biodiversidad). (2016). Estrategia nacional sobre biodiversidad de México y plan de acción 2016 2030. Ciudad de México: Comisión Nacional para el Conocimiento y Uso de la Biodiversidad.

CONAFOR (Comisión Nacional Forestal). (2010). Inventario nacional forestal y de suelos México 2004-2009 (Reporte Técnico). Jalisco, México: Comisión Nacional Forestal.

Crowther, T. W., Glick, H. B., Covey, K. R., Bettigole, C., Maynard, D. S., Thomas, S. M.,. . . Bradford, M. A. (2015). Mapping tree density at a global scale. Nature, 525(7568), 201-205.

Delaney, M., Brown, S., \& Powell, M. (1999). Carbonoffset report for the Noel Kempff climate action project, Bolivia (Reporte Técnico). Airlington, VA: Nature Conservancy.

Derroire, G., Balvanera, P., Castellanos-Castro, C., Decocq, G., Kennard, D. K., Lebrijatrejos, E., ... Healey, J. R. (2016). Resilience of tropical dry forests - a meta analysis of changes in species diversity and composition during secondary succession. Oikos, $125,1386-1397$

Dupuy, J. M., Hernández-Stefanoni, J. L., HernándezJuárez, R., Tetetla-Rangel, E., López-Martínez, J. O., Leyequién-Abarca, E., ... May-Pat, F. (2012). Patterns and correlates of tropical dry forest structure and composition in a highly replicated chronosequence in Yucatan, Mexico. Biotropica, 44(2), 151-162.

Egler, F. (1954). Vegetation science concepts. Initial floristic composition, a factor in old-field vegetation development. Vegetation, 4, 412-417.

ENAIPROS (Estrategia Nacional de Incremento a la Producción Sustentable). (2013). Estrategia nacional de manejo forestal sustentable para el incremento de la producción y productividad. 2013-2018 (Reporte Técnico). Jalisco, México: Comisión Nacional Forestal.

EEA (European Environment Agency). (2014). Terrestral habitat mapping in Europe: an overview (Reporte Técnico). Copenhagen, Denmark: Muséum National d'Histoire Naturelle- European Environment Agency. Recuperado de https://www.eea.europa.eu/ publications/terrestrial-habitat-mapping-in-europe.

Finegan, B. (1996). Pattern and process in neotropical secondary rain forests: The first 100 years of succession. Trends in Ecology \& Evolution, 11, 119-124.

Flashenberg, H., \& Galleti, H. A. (1999). El manejo forestal de la selva en Quintana Roo, México. In La selva maya, conservación y desarrollo. México: Siglo XXI.

Fox, J., Weisberg, S., Price, B., Adler, D., Bates, D., Baud-Bovy, G., . . . Winsemius, D. (2018). Companion to Applied Regression: car. R package version 3.0-0. Recuperado de https:// cran.r-project.org/ package $=$ car

Fujiki, S., Okada, K. I., Nishio, S., \& Kitayama, K. (2016). Estimation of the stand ages of tropical secondary forests after shifting cultivation based on the combination of WorldView-2 and time-series Landsat images. ISPRS Journal of Photogrammetry and Remote Sensing, 119(11), 280-293.

Gallardo-Cruz, J. A., Hernández-Stefanoni, J. L., Moser, D., Martínez-Yrizar, A., Llobet, S., \& Meave, J. (2018). Relating species richness to the structure of continuous landscapes: alternative methodological approaches. Ecosphere, 9(5), 1-15.

Gallardo-Cruz, J. A., Meave, J. A., González, E. J., LebrijaTrejos, E. E., Romero-Romero, M. A., Pérez-García, E. A., . . . Martorell, C. (2012). Predicting tropical dry forest successional attributes from space: Is the key hidden in image texture? PLOS ONE, 7(2), 38-45.

García Enriqueta (1990). Climas, 1: 4000 000. IV.4.10. Atlas Nacional de México (Vol. II). México: Instituto de Geografía, UNAM.

Garshelis, D. L. (2000). Delusions in habitat evaluation: Measuring use, selection, and importance. In L. Boitani \& T. K. Fuller (Eds.), Research techniques in animal ecology, controversies and consequences (pp. 111-164). New York: Columbia University. 
Gei, M., Rozendaal, D. M. A., Poorter, L., Bongers, F., Sprent, J. I., Garner, M. D., . . P Powers, J. S. (2018). Legume abundance along successional and rainfall gradients in neotropical forests. Nature Ecology \& Evolution, 2(5), 1-10.

George-Chacón, S. P. (2017). Modelización de la diversidad de especies de plantas leñosas en bosques tropicales secos mediante imágenes de alta resolución y datos LiDAR (Tesis de Maestría). Centro de Investigación Científica de Yucatán, A. C., México.

Gómez-Pompa, A. (1987). On Maya Silviculture. Estudios mexicanos, 3(1), 1-17.

Granados-Victorino, R. L., Sánchez-González, A., Martínez-Cabrera, D., \& Octavio-Aguilar, P., (2017). Estructura y composición arbórea de tres estadios sucesionales de selva mediana subperennifolia del municipio de Huautla, Hidalgo, México. Revista Mexicana de Biodiversidad, 88(1), 122-135.

Guevara-Sada, S., Laborde-Dovalí, J., \& Sánchez-Ríos, G. (2005). Los árboles que la selva dejó atrás. Interciencia, 30(10), 595-601.

Gutiérrez-Granados, G., Pérez-Salicrup, D., \& Dirzo, R. (2011). Differential diameter size effects of forest management on tree species richness and community structure: Implications for conservation. Biodiversity Conservation, 20, 1571-1585.

Hakkenberg, C. R., Peet, R. K., Urban, D. L., \& Song, C. (2018a). Modeling plant composition as community continua in a forest landscape with LiDAR and hyperspectral remote sensing. Environmental Applications, 28(1), 177-190.

Hakkenberg, C. R., Zhu, K., Peet, R. K., \& Song, C. (2018b). Mapping multi-scale vascular plant richness in a forest landscape with integrated LiDAR and hyperspectral remote sensing. Ecology, 99(2), 474-487.

Hernández-Stefanoni, J. L., Dupuy, J. M., Johnson, K. D., Birdsey, R., Tun-Dzul, F., Peduzzi, A., . . . LópezMerlín, D. (2014). Improving species diversity and biomass estimates of tropical dry forests using airborne LiDAR. Remote Sensing, 6, 4741-4763.

Hernández-Stefanoni, J. L., Dupuy, J. M., Tun-Dzul, F., \& May-Pat, F. (2010). Influence of landscape structure and stand age on species density and biomass of a tropical dry forest across spatial scales. Landscape Ecology, 26, 355-370

Ichter, J., Savio, L., Evans, D., \& Poncet, L. (2017). Stateof-the-art of vegetation mapping in Europe: results of a European survey and contribution to the French program CarHAB. Prodrome et cartographie des végétations de France, 6, 335-351.

Imai, N., Tanaka, A., Samejima, H., Baptist, J., Pereira, J. T., Titin, J., \& Kitayama, K. (2014). Tree community composition as an indicator in biodiversity monitoring of REDD +. Forest Ecology and Management, 313, 169-179.

Iqbala, M., Khanb, S. M., Khanc, M. A., Ahmadb, Z., \& Ahmadd, H. (2018). A novel approach to phytosociological classification of weeds flora of an agroecological system through cluster and indicator species analyses. Ecological Indicators, 84, 593-606.

Jardel-Peláez, E. J. (2015). Guía para la caracterización y clasificación de hábitats forestales. Jalisco, México: Comisión Nacional Forestal.

Kirk, D. A., Park, A. C., Smith, A. C., Howes, B. J., Prouse, B. K., Kyssa, N. G., \& Prior, K. A. (2018). Our use, misuse, and abandonment of a concept: Whither habitat? Ecology and Evolution, 8, 4197-4208.

Knipling, E. B. (1970). Physical and physiological basis for the reflectance of visible and near-infrared radiation from vegetation. Remote Sensing of Environment, 1, 155-159.

Korom, A., Mui-How, P., Wong, W., Matsuura, T., Saito, H., \& Hirata, Y. (2016). Exploring forest degradation: Tree density and aboveground biomass. Paper presented at the 37th Asian Conference on Remote Sensing, At Colombo, Sri Lanka.

Kruskal, J. B. (1964). Nonmetric multidimensional scaling. Psychometrika, 29(3), 1-27.

Lausch, A., Bannehr, L., Beckmann, M., Boehm, C., Feilhauer, H., Hacker, J. M., \& Rocchini, D. (2016). Linking earth observation and taxonomic, structural and functional biodiversity: Local to ecosystem perspectives. Ecological Indicators, 70, 317-339.

Legendre, P., \& Legendre, L. (1998). Numerical Ecology. Amsterdam: Elsevier.

Letcher, S. G., Lasky, J. R., Chazdon, R. L., Norden, N., Wright, J., Romero-Pérez, E., \& Williamson, G. B. (2015). Environmental gradients and the evolution of successional habitat specialization: a test case with 14 Neotropical forest sites. Journal of Ecology, 103, $1276-1290$

Liu, D., \& Xia, F. (2010). Assessing object-based classification: Advantages and limitations. Remote Sensing Letters, 1(4), 187-194.

Liu, Y., Gong, W., Hu, X., \& Gong, J. (2018). Forest type identification with random forest using Sentinel-1A, Sentinel-2A, multi-temporal Landsat-8 and DEM data. Remote Sensing, 10(949), 1-25.

Machala, M., \& Zejdová, L. (2017). Forest mapping through object-based image analysis of multispectral and LiDAR Aerial Data. European Journal of Remote Sensing, 47, 117-131. 
Martínez-Sánchez, J. L. (2016). Comparación de la diversidad estructural de una selva alta perennifolia y una mediana subperennifolia en Tabasco, México. Madera y Bosques, 22(2), 29-40.

McRoberts, R. E., Tomppo, E. O., \& Czaplewski, R. (2015). Sampling designs for national forest assessments. In knowledge reference for national forest assessments (pp. 23-40). Rome: Food and Agriculture Organization of the United Nations.

Miles, L., Newton, A., Defries, R., Ravilious, C., May, I., Blyth, S., \& Gordon, J. (2006). A global overview of the conservation status of tropical dry forests. Journal of Biogeography, 33, 491-505.

Mueller-Dombois, D., \& Ellenberg, H. (1974). Aims and methods of vegetation ecology. New York, USA: John Wiley and Sons.

Oksanen, J., Blanchet, F. G., Friendly, M., Kindt, R., Legendre, P., Mcglinn, D., \& Wagner, H. (2018). Vegan: Community ecology package. $\mathrm{R}$ package version 2.5.1. Recuperado de https://github.com/ vegandevs/vegan

Pasher, J., \& King, D. J. (2010). Multivariate forest structure modelling and mapping using high resolution airborne imagery and topographic information. Remote Sensing of Environment, 114(8), 1718-1732.

Pereira, H. M., Ferrier, S., Walters, M., Geller, G. N., Jongman, R. H., Scholes, R. J., \& Wegmann, M. (2013). Essential biodiversity variables. Science, 339(6117), 277-278.

Planet. (2016). RapidEye TM. Imagery product specifications (Tech. Rep.). Brandenburg, Germany: Blackbridge Rapideye.

Prieto, P. V., Seger, G. D. S., Sánchez-Tapia, A., Sansevero, J. B. B., Braga, J. M. A., \& Rodrigues, P. J. F. P. (2017). Secondary succession and fire disturbance promote dominance of a late-diverging tree lineage in a lowland neotropical forest. Plant Ecology \& Diversity, 10(4), 311-322.

R Core Team. (2016). R: A Language and environment for statistical computing. Vienna, Austria: R Foundation for Statistical Computing. Recuperado de https:// www.r-project.org/

Ripley, B., \& Venables, W. (2016). nnet: Feed-forward neutral networks and multinomial log-linear models. $\mathrm{R}$ package version 7.3-12. Recuperado de http://www. stats.ox.ac.uk/pub/MASS4/NeedsCompilation.

Román-Dañobeytia, F. J., Levy-Tacher, S. I., MacarioMendoza, P., \& Zúñiga-Morales, J. (2014). Redefining secondary forests in the Mexican forest code: Implications for management, restoration, and conservation. Forests, 5, 978-991.
Sánchez-Sánchez, O., Islebe, G. A., \& Valdez Hernández, M. (2007). Flora arbórea y caracterización de gremios ecológicos en distintos estados sucesionales de la selva mediana de Quintana Roo. Foresta Veracruzana, 9(2), 17-26.

Sánchez-Santos, G., Arreola-Palacios, J. A., López-Merlín, D., Maldonado-Montero, V., Olguín-Álvarez, M., Carrillo-Negrete, O., \& Puc-Kauil, R. (2015). Sitio de monitoreo intensivo del Carbono en Quintana Roo (Reporte Técnico). México: Comisión Nacional Forestal.

Slik, J. W. F., Arroyo-Rodríguez, V., Aiba, S. I., ÁlvarezLoayza, P., Alves, L. F., Ashton, P., \& Zang, R. (2015). An estimate of the number of tropical tree species. Proceedings of the National Academy of Sciences of the United States of America, 112(24), 7472-7477.

Solórzano, J., Meave, J. A., Gallardo-Cruz, A., González, E. J., \& Hernández-Stefanoni, J. L. (2017). Predicting old-growth tropical forest attributes from very high resolution (VHR) derived surface metrics. International Journal of Remote Sensing, 38(2), 492-513.

Spies, T. (1998). Forest Structure: A Key to the Ecosystem. Northwest Scientific Association, 72(2), 34-39.

Sterenczak, K., Lisanczuk, M., \& Erfanifard, Y. (2018). Delineation of homogeneous forest patches using combination of field measurements and LiDAR point clouds as a reliable reference for evaluation of lowresolution global satellite data. Forest Ecosystems, $5(1), 1-12$.

The Plant List. (2013). A working list of all known plant species, version 1. Recuperado de www.theplantlist. $\operatorname{org} / 1 /$.

Trimble. (2014). eCognition developer 9.0. Reference book. München, Germany: Trimble Germany GmbH.

Tropicos. (2013). Missouri Botanical Garden electronic databases. Recuperado de https://www.tropicos.org/

Urquiza-Haas, T., Dolman, P. M., \& Peres, C. A. (2007). Regional scale variation in forest structure and biomass in the Yucatan Peninsula, Mexico: Effects of forest disturbance. Forest Ecology and Management, $247,80-90$

Venables, W. N., \& Ripley, B. D. (2002). Modern applied statistics with $S$ (Fourth ed.). New York: Springer.

Wiggins, H. L. (2017). The influence of tree height on LiDAR's ability to accurately characterize forest structure and spatial pattern across reference landscapes (Tésis de Maestría). University of Montana, Missoula, EE.UU.

Zhang, Y., \& Chen, H. Y. H. (2015). Individual size inequality links forest diversity and above-ground biomass. Journal of Ecology, 103, 1245-1252. 